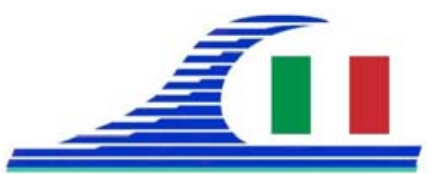

Conférence Méditerranéenne Côtière et Maritime EDITION 3, FERRARA, ITALIA (2015)

Coastal and Maritime Mediterranean Conference

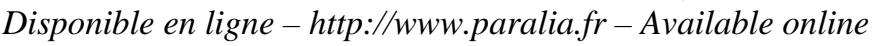

\title{
Détermination dynamique de la production naturelle des aérosols marins côtiers en Méditerranée occidentale
}

\author{
Rabia MERROUCHI ${ }^{1}$, Soumia MOURDANE ${ }^{2}$
}

1. Direction de la Météorologie Nationale (Casablanca), Maroc.

rabia.merrouchi@gmail.com

2. Laboratoire de Calcul Scientifique en Mécanique, Faculté des sciences Ben M’sick

Casablanca, Maroc.mordanesoumia@yahoo.fr

\section{Résumé :}

La mer est la première source des aérosols atmosphériques naturels. La composition chimique, la concentration et la taille des aérosols marins font d'eux un moyen d'échange de chaleur et de matière entre l'atmosphère et l'océan.

Plusieurs fonctions sources ont été développées pour la détermination des flux des aérosols marins émis à l'interface air-mer et en particulier en zone côtière. Les fonctions de génération de PIAZZOLA et al. (2003) ainsi que celle de VIGNATI et al. (2001) ont été développées à base de mesures expérimentales en Méditerranée occidentale.

Afin de permettre un suivi dynamique de la production des aérosols marins côtiers un modèle a été développé permettant de coupler les modèles de circulation atmosphérique de fine échelle en exploitation à la Direction de la Météorologie Nationale aux fonctions sources précitées. Les résultats des simulations appliquées à une situation de vents forts ayant affecté la méditerranée occidentale ont montré la capacité du modèle développé à calculer les concentrations des aérosols produites lors de cette situation ainsi que les distributions en taille des spectres associés.

Mots-clés : Spectre d'aérosols marins côtiers, Fetch, Modèle atmosphérique de fine échelle, Fonction source, Modèle d'aérosol, AROME.

\section{Introduction}

L'aérosol marin est produit principalement par le spray océanique (la production secondaire d'aérosols marins par conversions gaz-particules n'est pas prise en considération dans la présente étude). Sous l'action des vagues déferlantes, des gouttelettes d'eau de grosse taille sont projetées dans l'atmosphère. Après évaporation de l'eau de mer, les sels marins solides se retrouvent dans l'atmosphère et constituent des aérosols. La composition chimique de ce type d'aérosol est proche de celle de l'eau de mer. On retrouve donc essentiellement du chlore et du sodium, mais aussi de grandes quantités de nitrates et de sulfates (MASCLET \& CACHIER, 1998). Le taux d'émission annuel de l'aérosol marin est d'environ $10^{9}$ tonnes ce qui représente plus de $42 \%$ de la masse totale des aérosols atmosphériques. L’impact des aérosols marins sur le climat est généralement admis avec une contribution de plus de 44\% de l'épaisseur optique des 
Côtes méditerranéennes menacées :

Risques et défis dans le contexte du changement climatique

aérosols à l'échelle du globe avec un forçage radiatif au sommet de l'atmosphère d'environ -1.51 à -5.03 Wm² selon le rapport du GIEC (2001).

Afin de suivre l'évolution de la concentration des aérosols marins et en particulier en zone côtière, un modèle a été réalisé assurant le couplage entre les modèles atmosphériques de fine échelle et les modèles de production des aérosols marins.

Les simulations réalisées ont consisté en un couplage porté sur une situation de vents très forts ayant affecté la méditerranée occidentale la journée du 19 janvier 2014.

\section{Matériels et méthodes}

\subsection{Les fonctions sources des aérosols marins côtiers}

La production des aérosols marins est proportionnelle à la fraction de couche moutonneuse (W\%). En effet, le nombre de particules de rayons $r$ produites par $\mathrm{m}^{2}$ de surface, par seconde et pour une incrémentation d'un $\mu \mathrm{m}$ de $r$ est déterminé comme indiqué dans l'équation 1 (MONAHAN et al., 1986) :

$\frac{d F}{d r}=W \tau^{-1} \frac{d E}{d r}$

$\tau$ est un temps constant caractérisant l'amortissement exponentiel de la couche moutonneuse (généralement $\tau=3.53 \mathrm{~s}$ ), $d E / d r$ est le nombre de gouttelettes par incrément du rayon produit lors de la phase d'amortissement pour une unité de surface de cette couche moutonneuse (exprimée en $\mathrm{m}^{-2} \mu \mathrm{m}^{-1}$ ).

Il est bien évident que la concentration des aérosols augmente avec la fraction de la couche moutonneuse et en conséquence une meilleure connaissance de cette fraction $W$ est d'une extrême importance pour la détermination des concentrations des aérosols marins.

La fonction source de production des aérosols marins est également exprimée comme une fonction de puissance du vent en surface (VIGNATI et al., 2001).

PIAZZOLA et al. (2002) propose une formulation combinant à la fois l'effet du vent de surface, de l'humidité relative de l'air et du fetch sur la base de mesures in situ prises dans la baie de Toulon sur la mer méditerranée.

2.2 Les modèles numériques de circulation atmosphérique de fine échelle:

Deux modèles numériques de circulation atmosphérique de fine échelle ont été utilisés:

a) Le modèle Al_Bachir de la Direction de la Météorologie Nationale est un modèle atmosphérique à aire limitée centré sur le Maroc bâti et développé en déclinaison du modèle ALADIN dans le cadre d'un consortium regroupant 23 pays. Ce modèle atmosphérique produit des prévisions numériques à une échelle fine et à court terme (3 jours) avec une résolution spatiale de $10 \mathrm{~km}$.

b) Le modèle AROME a été conçu pour améliorer la prévision à courte échéance des phénomènes dangereux tels que les fortes pluies méditerranéennes et les orages 
violents. Le modèle est opérationnel à la DMN avec une résolution spatiale de $2.5 \mathrm{~km}$ et il est couplé au modèle AL BACHIR pour la définition des conditions aux limites.

\subsection{Le modèle de production des aérosols COMAC}

Le code de calcul (COMAC : COastal Marine Aerosols Code) a été réalisé afin de permettre le couplage entre les modèles de production d'aérosol marins côtiers en Méditerranée occidentale (VIGNATI et al., 2001 ; PIAZZOLA et al., 2003) à n’importe quel model numérique de circulation atmosphérique produisant des sorties en format GRIB et en particulier les modèles AL_BACHIR et AROME.

En entrée du modèle COMAC, on trouve les valeurs en point de grille de la température en surface, de température de l'eau, de l'humidité relative en surface et du champ de vent en surface. Ces valeurs sont extraites des modèles AL_BACHIR et AROME.

En sortie, le modèle COMAC génère des fichiers de sorties sous forme de spectres de concentration des aérosols marins produits en n'importe quel point de la grille et de cartes graphiques représentant la distribution spatiale des paramètres définis par l'utilisateur (vent, humidité, température, concentrations...etc).

Les modèles de nuage étant en général des modèles en classes de tailles des particules d'aérosols (Bin microphysical cloud models), COMAC est configuré de manière à calculer les concentrations d'aérosol pour chaque classe de rayon défini dans le modèle de nuage fournissant ainsi une distribution en taille de ces concentrations qui vont constituer l'état initial pour le modèle de nuage en question.

\section{Résultats}

Les simulations effectuées ont porté sur la situation du 19 Janvier 2014 caractérisée par des vents forts ayant affecté la méditerranée occidentale et ont atteint plus de 51 nœuds (94 km/h et 10 beauforts) au détroit de Gibraltar et au large des côtes du Portugal. La Figure 1 illustre quelques résultats issus du modèle COMAC, pour la situation du 19 janvier 2014, matérialisant le couplage entre le modèle atmosphérique de fine échelle AL-BACHIR et le modèle d'aérosol basé sur la formulation de VIGNATI et al, 2001.

\section{Conclusion}

Compte tenu de la contribution majeure des aérosols marins dans l'étude du climat et de ses changements ainsi que sur les activités humaines en zone côtière, le développement d'outils tels que COMAC s'avère importante pour le suivi des concentrations d'aérosols marins produits en mer méditerranée et le long de ses côtes.

L'analyse des distributions spatiales des concentrations obtenues avec COMAC pour cette journée du 19 janvier 2014 à 02h00 montre que les concentrations les plus importantes sont obtenues pour les aérosols de taille submicronique et fine. Par ailleurs, la distribution horizontale de ces concentrations est identique à celle du vent qui est à 
Côtes méditerranéennes menacées :

Risques et défis dans le contexte du changement climatique

l'origine de leur production avec un maximum au cap Sao Vicente au sud du Portugal ainsi que le long des côtes méditerranéennes Ouest de l’Algérie.
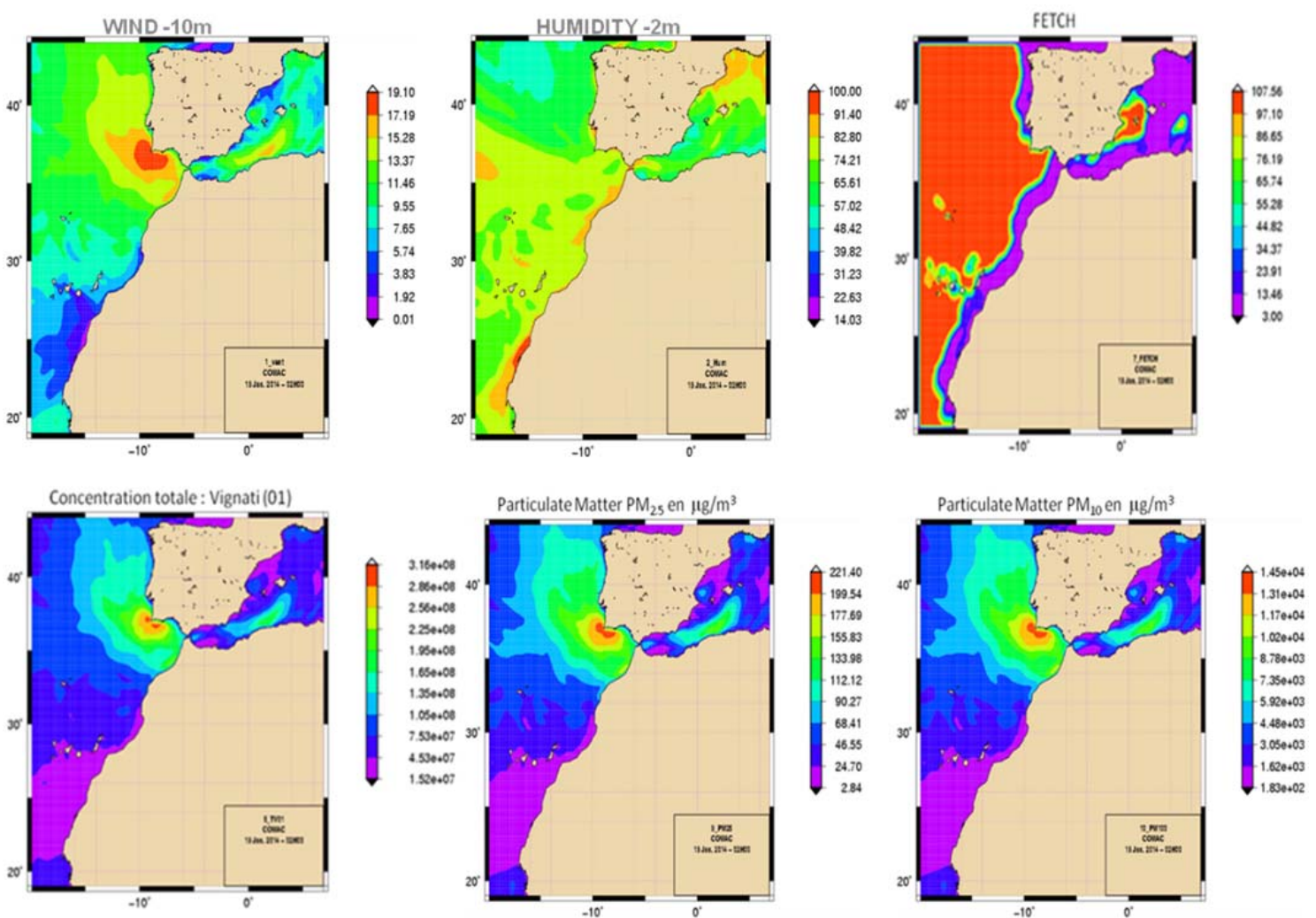

Figure 1. Distributions spatiales du vent en surface (à 10m), de l'humidité relative de l'air à $2 \mathrm{~m}$, du fetch et des concentrations totales $\left(\mathrm{cm}^{-3} \mu \mathrm{m}^{-1}\right)$ ainsi que des concentrations des particules en suspension $P M_{1}$ et $P M_{2.5}\left(\mu \mathrm{gm}^{-3}\right)$.

\section{Références bibliographiques}

MASCLET P., CACHIER H. (1998). L'aérosol atmosphérique. Caractéristiques physico-chimiques. Analusis magazine, Vol. 26, n 9, 6 p.

MONAHAN E.C., SPIEL D.E., DAVIDSON K.L. (1986). A model of marine aerosol generation via whitecaps and wave disruption. In Oceanic whitecaps and their role in air-sea exchange processes, Ed. by E.C. Monahan \& G.M. Niocaill, Springer, pp 167-174. http://dx.doi.org/10.1007/978-94-009-4668-2_16

PIAZZOLA J., FORGET P., DESPIAU S. (2002). A sea spray generation function for fetchlimited conditions. Ann. Geophys, Vol. 20, pp 121-131. http://dx.doi.org/10.5194/angeo-20-121-2002 VIGNATI E., DE-LEEUW G., BERKOWICZ R. (2001). Modeling coastal transport and effects of surf-produced aerosols on processes in marine atmospheric boundary layer. Journal of Geophysical Research, Vol. 106, pp 20.225-20.238. 